\title{
El rito de la «Apertura de la Boca» en el Antiguo Egipto: estudio bucodental de una cabeza egipcia momificada.
}

\author{
Labajo González, Elena, Perea Pérez, Bernardo, Dorado \\ Fernández, Enrique, Sánchez Sánchez, José Antonio y \\ Santiago Sáez, Andrés.
}

Cita:

Labajo González, Elena, Perea Pérez, Bernardo, Dorado Fernández, Enrique, Sánchez Sánchez, José Antonio y Santiago Sáez, Andrés (2014). El rito de la «Apertura de la Boca» en el Antiguo Egipto: estudio bucodental de una cabeza egipcia momificada. Revista Española de Medicina Legal, 40 (3), 116-119.

Dirección estable: https://www.aacademica.org/elenalabajogonzalez/21 ARK: https://n2t.net/ark:/13683/pcQr/fZn

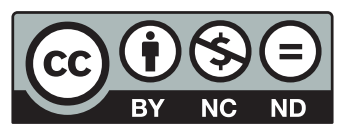




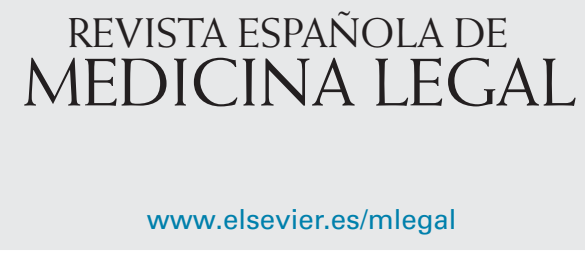

CASO MÉDICO-FORENSE

\title{
El rito de la «Apertura de la Boca» en el Antiguo Egipto: estudio bucodental de una cabeza egipcia momificada
}

\author{
Elena Labajo-González*, Bernardo Perea-Pérez, Enrique Dorado-Fernández, \\ José Antonio Sánchez-Sánchez y Andrés Santiago-Sáez
}

Escuela de Medicina Legal y Forense, Facultad de Medicina, Universidad Complutense de Madrid, Madrid, España

Recibido el 18 de septiembre de 2013; aceptado el 7 de octubre de 2013

\section{PALABRAS CLAVE}

Momia;

Embalsamamiento;

Ritual funerario;

Apertura de la Boca;

Antiguo Egipto

\section{KEYWORDS}

Mummy;

Embalming;

Unbandaged;

Funerary ritual;

Opening of the

Mouth;

Ancient Egypt
Resumen La momificación o embalsamamiento es un proceso que se desarrolló en el Antiguo Egipto para conservar el cuerpo tras la muerte, y preservar así la identidad del individuo en la vida futura, de acuerdo con sus costumbres funerarias.

El rito de la «Apertura de la Boca y los Ojos», formaba parte de los ritos funerarios y pretendía devolver al difunto la capacidad de hablar y de observar en el más allá. Se realizaba sobre el cadáver previamente a su embalsamamiento, o bien se «representaba» ante la momia ya embalsamada o una estatua de la misma.

Se presenta el caso de un cráneo humano momificado del Museo de Antropología Forense, Paleopatología y Criminalística de la Escuela de Medicina Legal de Madrid. Presenta protrusión lingual y fracturas en los dientes del grupo anterosuperior.

(c) 2013 Asociación Nacional de Médicos Forenses. Publicado por Elsevier España, S.L. Todos los derechos reservados.

The Ritual of «Opening of the Mouth» in Ancient Egypt: Study on a mummified egyptian head

Abstract Mummification or embalming is a process which was developed in Ancient Egypt in order to preserve the body after death, and thereby to maintain the individual's identity in the afterlife, in accordance with Ancient Egyptian funerary customs.

The ritual of Opening the Mouth and Eyes formed part of the funerary rituals and constituted an attempt to restore the ability of the deceased to speak and see in the life beyond. It was performed on the corpse prior to embalming, or it was «performed» in front of the mummy after embalming or a statue of the mummy.

\footnotetext{
* Autor para correspondencia.

Correo electrónico: elabajo@med.ucm.es (E. Labajo-González).
}

0377-4732/\$ - see front matter ๔ 2013 Asociación Nacional de Médicos Forenses. Publicado por Elsevier España, S.L. Todos los derechos reservados. http://dx.doi.org/10.1016/j.reml.2013.10.004 
We present the case of a mummified human cranium from the Forensic Anthropology, Paleopathology and Criminal Studies Museum at the Legal Medicine School of Madrid. It displays a protruding tongue and fractures on the teeth in the anterosuperior group.

(c) 2013 Asociación Nacional de Médicos Forenses. Published by Elsevier España, S.L. All rights reserved.

«He venido para abrazarte. Soy Horus. . Yo he presionado tu boca, yo soy tu hijo al que amas».

(Tumba tebana de Rekhmire. AN NIMO.)

\section{Introducción}

La momificación artificial, ritual o ceremonial, o embalsamamiento es un proceso que se desarrolló en el Antiguo Egipto para preservar el cuerpo de los faraones y otras figuras influyentes. La creencia en la inmortalidad del espíritu humano, según la mitología egipcia, originó que se practicara y desarrollara el embalsamamiento y la momificación, para poder preservar la identidad del individuo (Ka o fuerza vital) en la vida futura, según sus costumbres funerarias ${ }^{1,2}$.

Posteriormente, la práctica se extendió a todas las capas sociales. El cuerpo se preservaba de diferentes maneras: en general tras la extracción de los órganos internos, se procedía al vendado con vendas de lino e impregnado con diversas sustancias que conducían a la absorción del agua contenida en los tejidos humanos. Fue muy utilizado el natrón, natron o «sal divina», posiblemente el carbonato de potasio o sodio (mezcla de salitre y sosa) mezclado con otras sustancias (mirra, etc. $)^{1,2}$.

\section{El rito de la «Apertura de la Boca»}

El rito de la «Apertura de la Boca y los Ojos», es posiblemente una de las ceremonias más importantes del ritual funerario egipcio. Tuvo sus orígenes en el Imperio Antiguo (III-VI dinastías; hacia 2.640-2.155 a.C) y continuó a través del Imperio Medio, terminando al finalizar el Período Romano (30 a.C.-395 d.C). Con el se pretendía devolver al difunto la capacidad de hablar y de observar en el más allá ${ }^{-5}$.

La ceremonia de la «Apertura de la Boca», consistió en diferentes ritos que fueron evolucionando con el paso del tiempo. Según los estudios de Pahl (1984), si bien la ceremonia fue concebida en un primer momento como «acto simbólico» en el que se «animaba» una estatua representando el difunto o la momia del mismo, más tarde se llevó también a cabo directamente sobre la momia. Pese a la ausencia de fuentes literarias y pictóricas, las deformaciones postmortem de la región bucofacial observadas en numerosas momias, y la correlación entre dichas deformaciones, y el instrumental utilizado en la ceremonia, corroboran el hecho de que también se realizó la ceremonia de la «Apertura de la Boca» sobre cadáveres previamente o durante su embalsamamiento ${ }^{3-5}$.

En el rito de la «Apertura de la Boca», se utilizan diferentes instrumentos:

- Pesesh-kef: cuchillo en forma de cola de pez. Los ejemplares más antiguos estaban hechos de sílex.
Posteriormente se confeccionaron con obsidiana, vidrio o metal. Según la traducción, su nombre significaría «divisor de su espíritu ka».

- Ur-heaku: cuchillo en forma de serpiente. Los ejemplares más antiguos estaban hechos de sílex. Posteriormente se confeccionaron en jaspe, coralina y otras piedras semipreciosas. En ocasiones tienen inscrito el nombre del difunto. Según la traducción, su nombre significaría «grande en poderes».

- Mesjetyu y Nua: cuchillos en forma de azada. Normalmente se confeccionaban en madera.

- El doble dedo: cuchillo en forma de doble dedo. Aparece en los ritos funerarios del período tardío ${ }^{3,4}$.

Así pues, las menciones específicas en el Libro de la Apertura de la Boca, en el capítulo de la Ceremonia de Apertura de la Boca del Libro de los Muertos o las inscripciones halladas en diferentes tumbas («he presionado tu boca», «he presionado tu boca abierta», «tu boca se ha hecho firme», "he hecho que la forma de tu boca sea equilibrada con respecto a tus dientes» o «tus labios están hechos para ello; tu boca es hendida para ello»), pueden tener un significado tanto simbólico como práctico ${ }^{3-5}$.

\section{Estudio del rito de la «Apertura de la Boca» en el cráneo momificado ME-006 del Museo de Antropología Forense de la Universidad Complutense de Madrid}

La pieza objeto de estudio es una cabeza humana momificada del Museo de Antropología Forense, Paleopatología y Criminalística de la Universidad Complutense de Madrid (UCM). Está datada en la primera parte del Imperio Nuevo, de la XVIII dinastía, muy probablemente durante los reinados de Tutmosis IV (hacia 1.401-1.391 a.C) o Amenofis III (hacia 1.391-1.353 a.C), y su procedencia se sitúa en la necrópolis tebana. Los vendajes que presentaba la momia fueron retirados en el siglo XIX con fines científicos ${ }^{6}$.

La piel es de aspecto coriáceo, de color marrón oscuro, con pérdida de tejido en algunas zonas, dejando el hueso al descubierto. Conserva su cabello, de color marrón oscuro, ondulado, abundante y largo. Conserva asimismo ambos globos oculares, retraídos. Gran parte del apéndice nasal ha desaparecido, dejando al descubierto 2 objetos que obstruyen las fosas nasales. Las orejas se encuentran completas, con ambos conductos auditivos taponados. La boca está entreabierta y se observan los incisivos centrales y laterales, los caninos y los primeros premolares de la arcada superior. La lengua protruye ligeramente entre ambas arcadas (figs. 1 y 2). El cuello está presente hasta la altura de las 5 primeras vértebras cervicales ${ }^{6}$. 


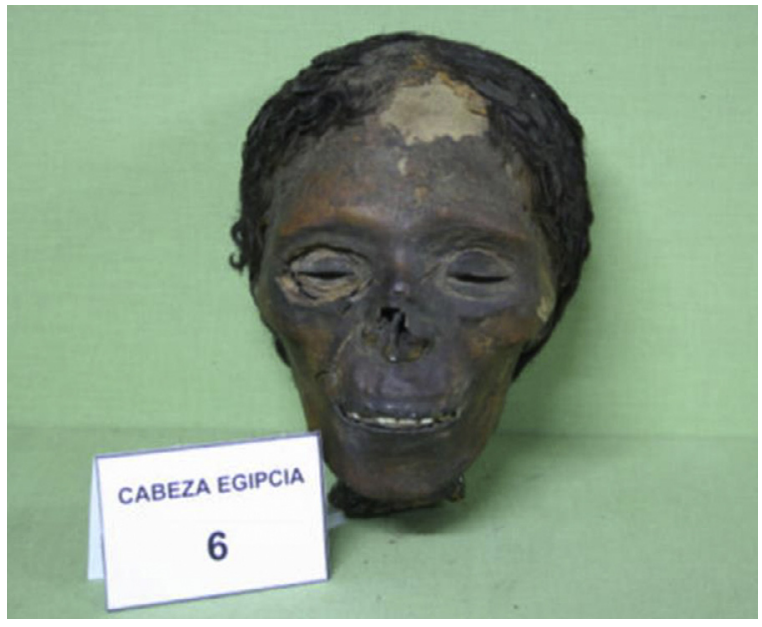

Figura 1 Cráneo momificado ME-006 del Museo de Antropología Forense de la UCM.

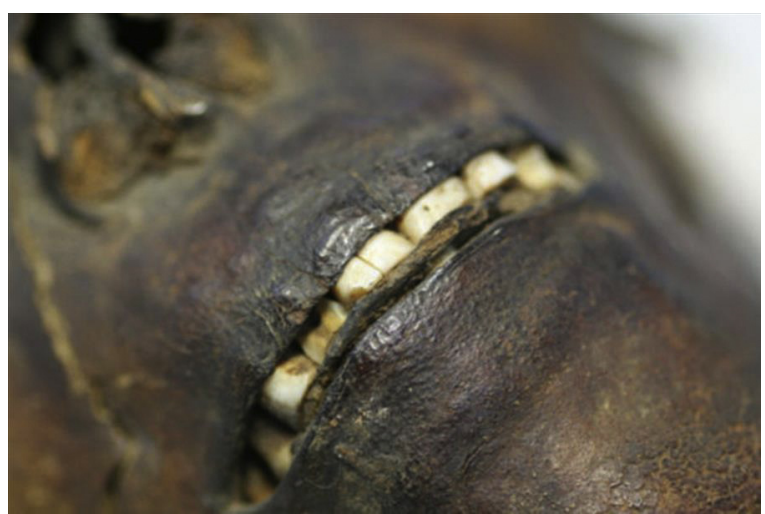

Figura 2 Cráneo momificado ME-006 del Museo de Antropología Forense de la UCM. Detalle.

Según al análisis antropométrico el cráneo corresponde a una mujer de unos 30-35 años de raza caucásica y variedad tipológica mediterránea (grácil) ${ }^{7-11}$.

El estudio radiográfico consiste en análisis de radiografías frontales, laterales de cráneo y ortopantomografías (figs. 3 y 4) mediante un generador de alta frecuencia y de

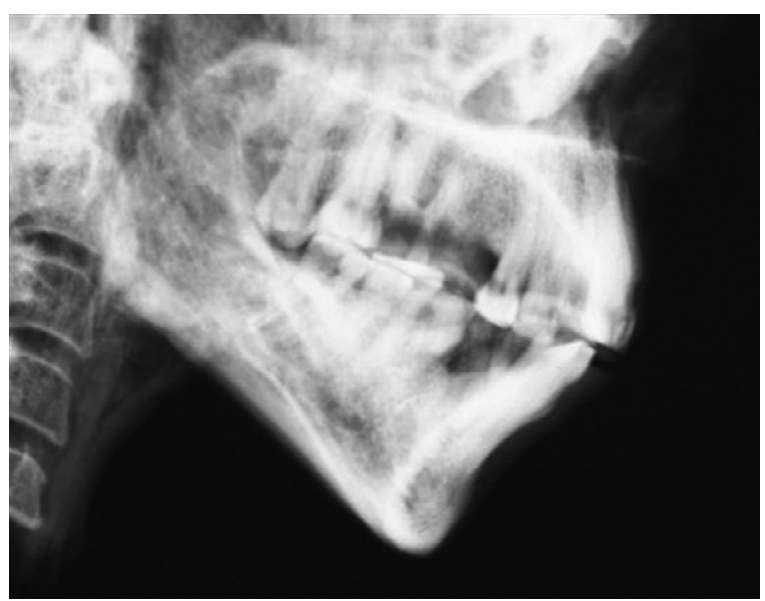

Figura 3 Cráneo momificado ME-006 del Museo de Antropología Forense de la UCM. Radiografía lateral de cráneo.

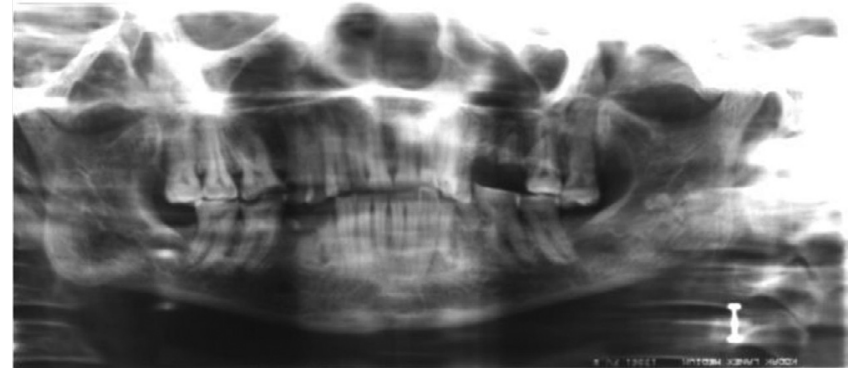

Figura 4 Cráneo momificado ME-006 del Museo de Antropología Forense de la UCM. Ortopantomografía.

potencial constante SHF de $50 \mathrm{Kw}$ de SEDECAL y sistema de rayos X panorámico General Electric GE3000 ${ }^{\circledR}$.

Para la nomenclatura dental se utilizó el sistema de la Federación Dental Internacional (FDI) o sistema dígito-dos. En este sistema de notación dentaria, la boca se divide en 4 cuadrantes correspondientes a las 4 hemiarcadas: 1 (superior derecho), 2 (superior izquierdo), 3 (inferior izquierdo), y 4 (inferior derecho). Los dientes de cada hemiarcada se numeran desde la línea media con números consecutivos del 1 al 8. Así, para nombrar un diente en concreto se utiliza un número de 2 cifras separadas por un punto. El primer número indica el cuadrante y el segundo la posición en la hemiarcada.

No se observan hipoplasias del esmalte ni presencia de tártaro. Se observa una caries interproximal, oclusodistal en el 1.5 que llega hasta la cavidad pulpar. El 1.1, el 2.1 y el 2.2 presentan una líneas de fractura verticales (postmortem). El 1.2 y 2.3 han perdido la cara vestibular por un traumatismo postmortem. El 2.5 está ausente por una pérdida antemortem. El 3.5 y el 4.4 son restos radiculares parcialmente reabsorbidos. El 3.4 presenta un granuloma periapical por afectación pulpar debido al desgaste dental. El 4.5 y el 4.8 están ausentes por sendas pérdidas antemortem. Según los grados de desgaste de Brabant ( $0=$ no hay desgaste; $1=$ desgaste parcial del esmalte; 2 = desgaste del esmalte dejando visible algunos puntos de dentina; 3 = desgaste total del esmalte, solo se ve la dentina, y 4 = desgaste de tola la corona hasta el cuello), el espécimen muestra un grado moderado de desgaste ${ }^{10-14}$ con el patrón que se describe a continuación:

Hemiarcada superior derecha:

18 (0); 17 (1); 16 (2); 15 (2); 14 (2); 13 (2); 12 (2); 11 (2). Hemiarcada superior izquierda:

28 (0); 27 (1); 26 (3); 25 (no); 24 (1); 23 (1); 22 (1); 21 (2). Hemiarcada inferior izquierda:

38 (no); 37 (2); 36 (2); 35 (2); 34 (2); 33 (2); 32 (2); 31 (2). Hemiarcada inferior derecha:

48 (no); 47 (2); 46 (2); 45 (3); 44 (no); 43 (no); 42 (2); 41 (2).

Las características del desgaste dental, así como su patrón de aparición concuerda con las técnicas de procesado de alimentos de la época (molienda de cereales en molinos y morteros de piedra, y la obtención de productos derivados de la harina con un contenido importante de partículas de piedra). La baja incidencia de caries y el estado bucodental, concuerdan con un acceso a una alimentación de calidad 
(aves, pescado, fruta,...), corroborado asimismo con el tipo de embalsamamiento que fue utilizado (propio de las clases sociales altas) ${ }^{15-17}$.

La protrusión lingual es muy corriente en las momias egipcias entre las que se ha practicado el rito de la «Apertura de la Boca». Las fracturas postmortem de los dientes del grupo anterosuperior, consistentes en líneas de fractura en $1.1,2.1$ y 2.2 , y la pérdida de la cara vestibular de 1.2 y 2.3 , corresponderían a traumatismos dentales postmortem debidos a dicha ceremonia ${ }^{3-6,18}$.

\section{Conclusión}

La ceremonia de la «Apertura de la Boca» fue una práctica ampliamente extendida en el Antiguo Egipto. En las primeras fases se «representaba» sobre una estatua que simbolizaba a la momia o al difunto, aunque más tarde pasó a realizarse directamente sobre la momia embalsamada o sobre los cadáveres previamente a su embalsamamiento. Consideramos que las alteraciones bucodentales del cráneo ME-006, del Museo de Antropología Forense, Paleopatología y Criminalística de la Facultad de Medicina de la UCM, corresponden a traumas dentales postmortem debidos al rito de la «Apertura de la Boca» en los cadáveres no embalsamados.

To be said: "The god Ptah shall open my mouth, and the god of my town shall unfasten the swathings, the swathings which are over my mouth. Thereupon shall come Thoth, who is equipped with words of power in great abundance, and shall untie the fetters, even the fetters of the god Set which are over my mouth. And the god Tem shall cast them back at those who would fetter me with them, and cast them at him. Then shall the god Shu open my mouth, and make an opening into my mouth with the same iron implement wherewith he opened the mouth of the gods. I am the goddess Sekhmet, and I take my seat upon the place by the side of Amt-ur the great wind of heaven. I am the great Stargoddess Saah, who dwelleth among the Souls of Anu. Now as concerning every spell, and every word which shall be spoken against me, every god of the Divine Company shall set himself in opposition thereto».

(Book of the Dead. Chapter of Opening the Mouth of the Osiris Ani. Anonimous)

\section{Conflicto de intereses}

Los autores declaran no tener ningún conflicto de intereses.

\section{Agradecimientos}

Queremos agradecer al Dr. D. Ángel Minaya Vaquero y al Dr. D. Ángel Minaya Bernedo, la ayuda prestada en la elaboración del estudio radiológico, así como a D. Ángel González de Arema la ayuda prestada en la documentación histórica y la datación.

\section{Bibliografía}

1. Kemp BJ. El antiguo Egipto. Anatomía de una civilización. Barcelona: Ed. Crítica; 1996.

2. Wallis Budge EA. The Egyptian book of the dead (the papyrus of Ani in the British Museum): Egyptian text, transliteration, and translation. New York: Dover Publications, Inc; 1967.

3. Wallis Budge EA. The book of opening the mouth. Reprint Edition. New York: Ayer Company Publishing INC; 1984.

4. Pahl WM. The ritual of opening the mouth. In science in Egyptology: Proceedings of the science in Egyptology Symposia. Manchester: Manchester University Press; 1986.

5. Forshaw RJ. The practice of dentistry in Ancient Egypt. Br Dent J. 2009:481-6.

6. González A, González M, Ruiz M, Minaya A, Sánchez JA, López O, et al. De Guinea a Egipto: redescubrimiento de una pieza egipcia antigua en el Museo Reverte. II Congrés de l'Associació Catalano-Balear de Paleopatologia. Barcelona, 7 de junio de 2008.

7. Greef CJ. Paleopathology: Signs and lesions in skeletal remains of epidemics and diseases of biblical times in Syro-Palestine. Tesis Doctoral. University of South Africa, 2005.

8. Hoffman H, Hudgins PA. Head and skull base features of nine Egyptian mummies. Am J Roent. 2002;178:1367-76.

9. Harris JE, Wente E. An X-ray atlas of the royal mummies. Chicago: Chicago University Press; 1980.

10. Campillo D, Subirá E. Antropología física para arqueólogos. Barcelona: Ed. Ariel; 2004.

11. Thekkaniyil JK, Bishara SE, James MA. Dental and skeletal findings on an ancient Egyptian mummy. Am J Orthod Dentof Orthop. 2000;117:10-4.

12. Peluso A. Dental conditions of Egyptian heads: adiological and direct examination. J Biol Res. Vol. LXXX, n. ${ }^{\circ}$ 1. Proceedings V World Congress on mummy studies. Turin, 2005. Vol. LXXX, n. ${ }^{\circ} 1$.

13. Miller J. Dental health in Ancient Egypt. J Biol Res. Proceedings V World Congress on mummy studies. Turin, 2005. Vol. XXX, n. ${ }^{\circ} 1$.

14. Hillson S. Dental anthropology. Cambridge: Cambridge University Press; 1996.

15. Molnar S. Human tooth wear, tooth function and cultural variability. Am J Phys Anthrop. 1971;34:175-90.

16. Teaford MF, Lytle JD. Brief communication: Diet induced changes in rates of human tooth microwear: A case study involving stone-ground maize. Am J Phys Anthrop. 1996;100: $143-7$.

17. Smith BH. Patterns of molar wear in hunter-gatherers and agriculturalists. Am J Phys Anthrop. 1984;63:39-56.

18. Robson $\mathrm{K}$, Wood $\mathrm{H}$. The utility of minimal ct scanning in the study of two Egyptian mummy heads. Int J Osteoarchaeol. 1999;9:199-204. 